\title{
Combination of long-acting HIV fusion inhibitor albuvirtide and LPV/r showed potent efficacy in HIV-1 patients
}

\author{
Hongwei Zhang ${ }^{1 \dagger}$, Ronghua Jin ${ }^{1 \dagger}$, Cheng Yao ${ }^{2}$, Tong Zhang ${ }^{1}$, Meixia Wang ${ }^{1}$, Wei Xia ${ }^{1}$, Haiyan Peng ${ }^{3}$,
} Xiaojuan Wang ${ }^{3}$, Rongjian $\mathrm{Lu}^{2}$, Changjin Wang ${ }^{2}$, Dong $\mathrm{Xie}^{2^{*}}$ and Hao $\mathrm{Wu}^{4^{*}}$

\begin{abstract}
Background: Long acting antiretroviral drugs represent a promising approach for chronic treatment of HIV infection. Here, we study the efficacy and safety of albuvirtide (ABT), an HIV-1 fusion inhibitor with a half life of 11-12 days in human.

Methods: ABT was evaluated in a 7-week, open-label and randomized trial, combining with LPV/r. Twenty HIV-1-infected adults were assigned to two dose groups, receiving ABT (160 or $320 \mathrm{mg}$ ) given weekly and LPV/r given twice daily.

Results: At week 7, the decline of HIV-1 RNA from baseline was 1.9 (1.3-2.3) $\log _{10}$ and $2.2(1.6-2.7) \log _{10} \mathrm{copies} / \mathrm{ml}$, and suppression of HIV-1 RNA to below 50 copies/ml was achieved in $11.1 \%(1 / 9)$ and $55.6 \%$ (5/9) patients, for the 160 and $320 \mathrm{mg}$ dose group respectively.
\end{abstract}

Conclusion: A clear dose-efficacy correlation of ABT was demonstrated. ABT combining with LPV/r is a promising two-drug regimen to be tested in larger patient population.

\section{Background}

Current antiretroviral therapy (ART) requires strict lifelong adherence to daily drug taking. Long-acting ART agents, capable of being administered on a weekly or less frequent basis, have the potential to improve adherence to therapy and allow a more forgiving time window of drug administration [1].

Albuvirtide (ABT) is a chemically modified peptide derived from the N-terminal sequence of HIV-1 gp41, and contains a 3-maleimimidopropionic acid (MPA) group in its 13th lysine side chain [2]. Previous studies demonstrated that upon intravenous injection ABT could rapidly conjugate with serum albumin and dramatically

*Correspondence: dxie@frontierbiotech.com; whdoc@sina.com

${ }^{+} \mathrm{H}$ ongwei Zhang and Ronghua Jin contributed equally to this work

2 Nanjing Frontier Biotechnologies Co. Ltd., 5 Qiande Road, Jiangning

District, 211122 Nanjing, People's Republic of China

${ }^{4}$ Center for Infectious Diseases, Beijing You'an Hospital, Capital Medical

University, 8 Xitoutiao, Youanmenwai St, Fengtai Dist, 100069 Beijing,

People's Republic of China

Full list of author information is available at the end of the article extend the peptide in vivo half-life from 1.7 to $25.8 \mathrm{~h}$ in rats and from 10.9 to $102.4 \mathrm{~h}$ in monkeys [2]. A recent study using pseudoviruses showed potent inhibitory activity of ABT against a broad spectrum of HIV-1 strains, including those commonly observed in China and some variants resistant to T20 [3]. A phase 1 single agent study in HIV-1 infected patients showed excellent safety profile of single and multiple dose of ABT, a halflife of 11-12 days, and a clear dose related antiviral activity [4].

In this study in naive HIV-1 patients, a novel two-drug regimen combining $\mathrm{ABT}$ and lopinavir/ritonavir (LPV/r) was tested, their drug-drug interaction investigated, and short-term safety and efficacy profiles characterized.

\section{Methods \\ Study population}

Antiretroviral treatment-naïve HIV-1-infected patients aged 18-50 years were eligible for this study if they had HIV RNA levels between 5000 and 1,000,000 copies/ 
$\mathrm{ml}, \mathrm{CD} 4$ cell counts more than 350 cells/ $\mu \mathrm{l}$, body weight more than $40 \mathrm{~kg}$ and body mass index (BMI) between 18.0 and $27.0 \mathrm{~kg} / \mathrm{m}$ [2]. They had a normal level of albumin and no severe liver and kidney damage, and had not received any antiretroviral therapy for HIV and HBV or stopped antiretroviral therapy for more than 6 months. Exclusion criteria included acute HIV infection, severe opportunistic infections and tumors, severe diseases of digestive tract, hematology, metabolism, psychology and heredity, previous treatment of other HIV fusion inhibitors, drug abuse, pregnancy, and breastfeeding. Female patients with child bearing potential and heterosexually active male patients were required to use effective contraception during the study. The study was approved by the institutional ethics committee of Beijing You'an Hospital, and all potential subjects provided written informed consent before undergoing procedures.

\section{Study design}

The study was a single-site, open-label, and randomized parallel study conducted in the Center for Infectious Diseases, Beijing You'an Hospital, Capital Medical University. Twenty antiretroviral treatment-naïve HIV-1 infected patients were enrolled and randomized into two dose groups (10 in each group) to receive ABT by intravenous infusion at dose levels of 160 or $320 \mathrm{mg}$, and $\mathrm{LPV} / \mathrm{r}(400 / 100 \mathrm{mg})$ twice daily. On Day 5-7, ABT was given daily for 3 days, then given weekly till Day 40; and $\mathrm{LPV} / \mathrm{r}$ was given from Day 1 to 46 . The subjects were followed for a 47-day observation period. After the study all participants were treated with triple combination antiviral therapy containing tenofovir, lamifudine and LPV/r.

The plasma HIV-1 RNA was measured using a branched-chain DNA method (bDNA, version 3.0, Bayer Healthcare LLC, Diagnostics Division, Tarrytown, NY) with a detection limit of 50 copies/ml. CD4 cell counts were measured using a FACS count system (FACS Calibur, Becton Dickinson, USA).

\section{Statistical analysis}

This was a pilot study without power calculations or sample size estimation. Data were presented as the mean $\pm S D$ if not specified. The efficacy and safety analyses were performed for the intent to treat (ITT) population, defined as all subjects who were enrolled in the trial and received at least one dose of study medication. All statistical analyses were performed using SAS version 9.2.

\section{Results}

Twenty antiretroviral treatment-naïve HIV-1 infected patients (Table 1) were enrolled and randomized into two dose groups (10 in each group) to receive ABT (160 or $320 \mathrm{mg}$ ) and LPV/r. There were no significant differences
Table 1 Baseline characteristics of subjects

\begin{tabular}{|c|c|c|}
\hline & $160 \mathrm{mg} \mathrm{ABT}+\mathrm{LPV} / \mathrm{r}$ & $320 \mathrm{mg} \mathrm{ABT}+\mathrm{LPV} / \mathrm{r}$ \\
\hline \multicolumn{2}{|c|}{ Randomized and treated 10} & 10 \\
\hline Excluded & 1 & 1 \\
\hline SS for safety analysis & 10 & 10 \\
\hline ITT for efficacy analysis & 9 & 9 \\
\hline Sex (male/female) & $8 / 2$ & $7 / 3$ \\
\hline Race (Han/other) & $9 / 1$ & $10 / 0$ \\
\hline Age (years) & $31.9(18 \sim 48)$ & $37.4(24 \sim 47)$ \\
\hline Body weight (kg) & $67.8(53 \sim 88)$ & $65.4(53 \sim 76)$ \\
\hline BMI $\left(\mathrm{kg} / \mathrm{m}^{2}\right)$ & $22.8(19 \sim 27)$ & $22.8(18 \sim 26)$ \\
\hline $\begin{array}{l}\text { Baseline HIV-1 RNA } \\
\left(\log _{10} \text { copies/ml) }\right.\end{array}$ & $4.27(3.53 \sim 4.70)$ & $4.27(3.32 \sim 5.14)$ \\
\hline Baseline CD4 (cell/ul) & $517.0(350 \sim 774)$ & $566.6(350 \sim 1070)$ \\
\hline
\end{tabular}

Data are presented as $\mathrm{n}$ or mean (range)

in age, body weight, HIV viral load and CD4 cell count at baseline between the two groups. Nineteen subjects completed the study with one withdrawal due to lost to follow-up. Two patients whose baseline HIV viral load did not meet the inclusion criteria and their viral load data were excluded from efficacy analysis. All 20 subjects were included in the pharmacokinetic and safety analysis.

From Day 14 till the end of the study, the viral load (plasma HIV-1 RNA) of all patients decreased $>1.0 \log _{10}$ copies $/ \mathrm{ml}$. At the 47 th day, the mean viral load decreased from baseline was $1.91 \pm 0.36 \log _{10}$ and $2.20 \pm 0.33 \log _{10}$ copies $/ \mathrm{ml}$ for the 160 and $320 \mathrm{mg}$ groups, respectively (Fig. 1a). The percentage of subjects with viral load $<50$ copies $/ \mathrm{ml}$ was $11.1 \%$ in the $160 \mathrm{mg}$ group and $55.6 \%$ in the $320 \mathrm{mg}$ group (Fig. 1b). Pharmacokinetic/pharmacodynamic (PK/PD) relationship analysis showed that the decrease of HIV viral load was positively correlated with $\mathrm{AUC}_{0-168 \mathrm{~h}}, \mathrm{C}_{\text {min }}$ and $\mathrm{C}_{\text {trough }}$ of ABT. After treatment with $\mathrm{ABT}$ and $\mathrm{LPV} / \mathrm{r}$ for 47 days, the mean absolute $\mathrm{CD} 4^{+}$ cell count change relative to baseline was -5 cells $/ \mu$ for the $160 \mathrm{mg}$ group and 52 cells $/ \mu \mathrm{l}$ for the $320 \mathrm{mg}$ group respectively.

The pharmacokinetic profile of co-administration of $\mathrm{ABT}$ and LPV/r showed weak or no interaction. The results will be reported elsewhere. There were no serious adverse events during the 47 days of treatment. Eight cases of adverse events were observed in 7 subjects in $160 \mathrm{mg}$ group and nine cases in 8 subjects in $320 \mathrm{mg}$ group, but only six cases were related to investigational regimen in each group. All adverse events were mild, mainly triglycerides level elevation, diarrhea, nausea and skin rash. No injection site reactions were found during the trial.

\section{Discussion}

In chronic management of HIV infection with ART, one of the most challenges is poor patient adherence to 

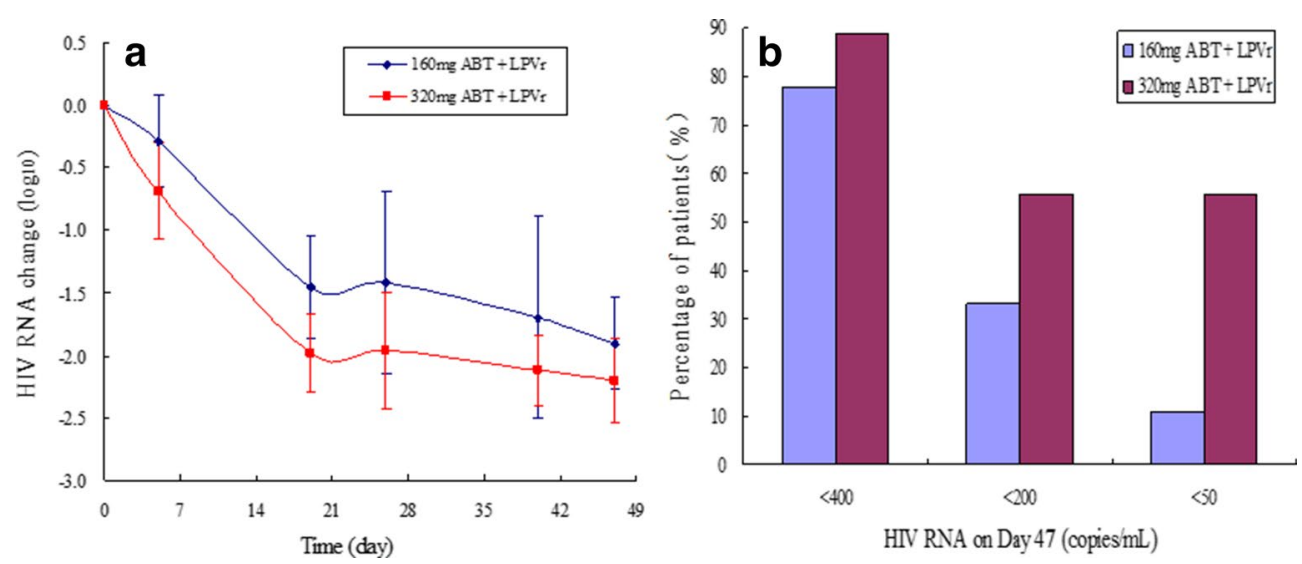

Fig. 1 Anti-HIV activity of albuvirtide and LPV/r. a Mean change of plasma HIV RNA over time by dose group. b Percentage of patients with viral load <50, 200 and 400 copies/ml on Day 47

treatment, which often results in treatment failure and emergence of drug resistance [5]. ABT is an experimental anti-HIV peptide targeting the HIV-1 envelop protein gp-41 at an area that's different from that of T20. The 13th residue of ABT contains a MPA modification that allows ABT to react with serum albumin. This extends its half-life to 11-12 days in human while retaining anti-HIV activity [3]. Although long-acting AIDS drugs are much sought after, how to administer such molecules with current oral drugs remain to be studied.

In this phase 2 trial, we designed a two-drug regimen that includes a weekly given $\mathrm{ABT}$ and twice daily given $\mathrm{LPV} / \mathrm{r}$. It represents the first attempt to combine an approved, orally taken ART with a long-acting, injectable anti-HIV agent to treat HIV infected patients. For 47 days, the novel regimen was safe and exhibited potent anti-HIV activity. All treated patients, including one whose baseline viral load was $>5 \log _{10}$ copies $/ \mathrm{ml}$, showed $>1 \log _{10}$ copies $/ \mathrm{ml}$ reduction of viral load. Importantly, data of the $320 \mathrm{mg}$ group showed a trend of superior anti-HIV activity to that of the $160 \mathrm{mg}$ group. This demonstrates clear contribution of ABT to the plasma HIV RNA reduction in the two-drug regimen.

ABT is the first long-acting antiretroviral drug developing in China. With the limitation of no long-acting drugs combined with ABT, we also chose LPV/r for the ART regimen in the phase III clinical trial, a 48-week, randomized, controlled, open-label, multicenter study to investigate the safety and efficacy of ABT, which may have potential as next-generation HIV fusion inhibitors targeting gp41 for clinical use [6].

\section{Conclusion}

In summary, this 7-week study shows that ABT combined with LPV/r is safe and effective. Compared with regimens of 3-4 drugs, the two-drug regimen could offer a simplified therapy with better safety and less drug-drug interaction. The long half-life of ABT potentially allows a 3-day window for weekly administration and is more forgiving in adherence than daily taken drugs.

\section{Authors' contributions}

Designed the study: DX and HW; conducted the study: RJ, TZ, MW, HZ, WX, R L, CW, CY, HP and XW; analyzed the data: DX, HW, CY and R J; wrote the manuscript: HZ, CY, HW and DX. All authors read and approved the final manuscript.

\section{Author details}

${ }^{1}$ Beijing You'an Hospital, Capital Medical University, 100069 Beijing, People's Republic of China. ${ }^{2}$ Nanjing Frontier Biotechnologies Co. Ltd., 5 Qiande Road, Jiangning District, 211122 Nanjing, People's Republic of China. ${ }^{3}$ Beijing Co-CRO Medical Development Co. Ltd., Beijing, People's Republic of China.

${ }^{4}$ Center for Infectious Diseases, Beijing You'an Hospital, Capital Medical University, 8 Xitoutiao, Youanmenwai St, Fengtai Dist, 100069 Beijing, People's Republic of China.

\section{Acknowledgements}

The study was sponsored by Nanjing Frontier Biotechnologies Co., Ltd., and partly supported by the Beijing Municipal Science \& Technology Commission (D131100005313005), the Ministry of Science and Technology of China (Grant No. D141100000314005, D141100000314002), the national "twelfth five" major projects of significant new drugs development (Grant No. 2013ZX09101001), and the national "twelfth five" major projects of AIDS and viral hepatitis and other major infectious diseases prevention and control (Grant No. 2012ZX10001-003). The study was registered at http://www.chictr.org/, \# ChiCTR-TRC-13003140.

\section{Competing interests}

C.Y., R.L., C.W. and D.X. are employees of Nanjing Frontier Biotechnologies Co., and hold stock in the company. All other authors declare that they have no competing interests.

Received: 30 October 2015 Accepted: 26 January 2016

Published online: 10 February 2016

\section{References}

1. Dolgin E. Long-acting HIV drugs advanced to overcome adherence challenge. Nat Med. 2014;20(4):323-4. 
2. Xie D, Yao C, Wang L, Min W, Xu J, Xiao J, et al. An albumin-conjugated peptide exhibits potent anti-HIV activity and long in vivo half-life. Antimicrob Agents Chemother. 2010;54(1):191-6.

3. Chong H, Yao X, Zhang C, Cai L, Cui S, Wang Y, et al. Biophysical property and broad anti-HIV activity of albuvirtide, a 3-maleimimidopropionic acid-modified peptide fusion inhibitor. PLoS ONE. 2012;7(3):e32599.

4. Wu H, Yao C, Lu R, Zhang T, Wang M, Zhao H, et al. Albuvirtide, the first long-acting HIV-1 fusion inhibitor, suppressed viral replication in HIVinfected adults.In: The Inter-science Conference on Antimicrobial Agents and Chemotherapy (ICAAC), San Francisco, USA; Sep 9-12, 2012. Paper $\mathrm{H}-554$.

5. Cadosch D, Bonhoeffer S, Kouyos R. Assessing the impact of adherence to anti-retroviral therapy on treatment failure and resistance evolution in HIV. J R Soc Interface. 2012;9(74):2309-20.

6. Zhang D, Li W, Jiang S. Peptide fusion inhibitors targeting the HIV-1 gp41: a patent review (2009-2014). Expert Opin Ther Pat. 2015;25(2):159-73.
Submit your next manuscript to BioMed Central and we will help you at every step:

- We accept pre-submission inquiries

- Our selector tool helps you to find the most relevant journal

- We provide round the clock customer support

- Convenient online submission

- Thorough peer review

- Inclusion in PubMed and all major indexing services

- Maximum visibility for your research

Submit your manuscript at www.biomedcentral.com/submit
() Biomed Central 